\title{
Interacción entre la pesquería del bacalao de profundidad, Dissostichus eleginoides (Nototheniidae), con el cachalote y la orca en el sur de Chile: revisión del estado de conocimiento
}

Interaction between the patagonian toothfish fishery, Dissostichus eleginoides (Nototheniidae), with the sperm whale and killer whale off southern Chile: review of the knowledge status

Benjamín Cáceres ${ }^{1}$, Anelio Aguayo-Lobo ${ }^{1,2}$ \& Jorge Acevedo ${ }^{3}$

\section{Resumen}

Interacciones entre la pesquería de palangre del bacalao de profundidad (Dissostichus eleginoides) y cetáceos en el Océano Austral se han registrado desde hace más de tres décadas. Las dos especies de cetáceos que se han visto mayormente involucradas son el cachalote (Physeter macrocephalus) y la orca (Orcinus orca). Ambas especies han sido observadas depredando sobre las líneas de palangre y las pesquerías de bacalao se han visto sustancialmente afectadas por la notoria disminución de las capturas. En este trabajo, resumimos el conocimiento que se tiene de las interacciones al sur de los $47^{\circ} \mathrm{S}$ en buques pesqueros industriales. Actualmente, las interacciones ocurren principalmente con la orca y en un menor grado con el cachalote, luego de la modificación del arte de pesca que ha disminuido la depredación de este último. A la fecha, distintas alternativas para disminuir esta interacción han sido probadas, incluyendo modificaciones físicas del arte de pesca, dispositivos acústicos y cambios en las prácticas pesqueras, sin embargo, no existe una solución a este problema. A futuro, un conjunto de acciones deberían ser coordinadas efectivamente entre los operadores pesqueros, investigadores, ingenieros pesqueros e instituciones públicas del estado, a través de un programa colaborativo con el objetivo de disminuir estas interacciones.

\section{Palabras clave:}

Océano Austral, pesquerías, Physeter macrocephalus, Orcinus orca.

\section{Abstract}

Interactions between the longline toothfish (Dissostichus eleginoides) fisheries and cetaceans in the Southern Ocean have been registered over three decades. Two species primarily involved in these interactions are the sperm whale (Physeter macrocephalus) and the orca (Orcinus orca). Both species have been registered depredating on the longlines and fisheries have been substantially affected by the noticeable decrease on the catch. In this review, we summarized current knowledge about these interactions occurring south of $47^{\circ} \mathrm{S}$ in the industrial fleet. At present, interactions occur predominantly with the orca and in a lower extent with the sperm whale, after the modification of the fishing gear that diminished the depredation of the sperm whale. To date, many alternatives have been tested to repel cetaceans, including physical modifications of the fishing gear, acoustic devices, and changes in the fishing practices by the vessels, yet none seem effective. In the future, not one solution but a complex mix of actions must be coordinated effectively between the fishing operators, researchers, fishery engineers and

Asociación de Investigadores del Museo de Historia Natural (ASIMHIN) Río Seco, Punta Arenas, Chile.

$\checkmark$ benjamincaceresm@gmail.com

2 Instituto Antártico Chileno, Punta Arenas, Chile. aaguayo@ inach.cl

3 Centro de Estudios del Cuaternario Fuego-Patagonia y Antártica, Punta Arenas, Chile. j.acevedo@cequa.cl 
State Public Institutions in order to diminish these interactions.

\section{Key words:}

Southern Ocean, fisheries, Physeter macrocephalus, Orcinus orca.

\section{INTRODUCCIÓN}

Chile es un importante país pesquero del mundo debido a la alta productividad de su mar territorial, albergando especies de gran importancia comercial que son explotadas por una variedad de pesquerías nacionales. Así mismo, las aguas jurisdiccionales del país poseen una alta diversidad de cetáceos informándose de 43 especies (AguayoLobo et al. 1998), las que representan el $49 \%$ del total conocidas a nivel mundial. Todas las especies de cetáceos en el país están protegidas desde el cese de las capturas comerciales en el año 1983, mediante la normativa legal del Decreto 225 del Ministerio de Economía Fomento y Reconstrucción del año 1995, Decreto 250 del mismo Ministerio del año 2008 y mediante la Ley 20.293 del mismo año (Aguayo et al. 2011).

Dado que las poblaciones de cetáceos y la actividad pesquera coinciden en las mismas áreas geográficas, las interacciones entre ambas son inevitables. Estas interacciones han sido documentadas desde hace más de tres décadas (Northridge, 1984; Salas et al. 1987; Ashford et al. 1996). Sin embargo, es importante comprender que las interacciones se producen entre el hombre (Pesquerías) y los cetáceos (Trites et al. 1997; Aguayo-Lobo, 1999). Comúnmente se reconocen dos tipos de interacciones entre las pesquerías y los cetáceos, las que son clasificadas como Ecológicas y Operacionales (Northridge \& Hofman, 1999). Las primeras, implican una competencia alimentaria por un mismo recurso pesquero (desde el punto de vista del hombre) o de las presas (desde el punto de vista de cetáceos), y la consecuente sobreposición del hábitat para ambos contendores. Las interacciones operacionales son de carácter técnico y se manifiestan mientras se realizan las faenas extractivas y producen como consecuencia una pérdida económica para el sector pesquero (e.g., remoción de la pesca desde los aparejos y la posible pérdida del arte de pesca debido a enmallamientos incidentales de cetáceos) y la obtención de alimento para los cetáceos que en otras circunstancias habría sido poco factible de conseguir. No obstante, estás interacciones pueden poner al mismo tiempo en riesgo la vida de los animales dado que puede aumentar las posibilidades de recibir lesiones o terminar enmallados, con la consiguiente muerte del animal (Angliss \& DeMaster, 1997).

Entre las pesquerías nacionales, y en particular en la región sur-austral, la pesquería del bacalao de profundidad, Dissostichus eleginoides (Smith, 1958), es una importante actividad pesquera iniciada como exploratoria durante 1955 (González, 1962) y extendida ya en la década de 1990 (Lemaitre et al. 1991). Este pez nototénido catalogado como abiso-bentónico (Pavéz et al. 1983) y/o bento-demersal (Salas et al. 1987), por el Pacífico se extiende a lo largo del talud continental desde Ecuador hasta las islas Diego Ramírez (Chile) e islas Shetland del Sur (Antártica). En el Atlántico, se extiende desde el sur de Brasil hasta Argentina, incluyendo las islas Falkland/ Malvinas, islas Georgias del Sur, Sándwich del Sur y Orcadas. En el Océano Índico, se encuentra en aguas de las islas Marion, Prince Edward, Crozet, Kerguelen, Heard, Amsterdam y San Paul (Salas et al. 1987; Trites et al. 1997; Arana \& Vega, 1999; González, 2003; Kaschner \& Pauly, 2005; Söffker et al. 2015).

Las interacciones entre la pesquería del bacalao y cetáceos ocurren en varias localidades del mundo, siendo las principales especies que interactuan la orca (Orcinus orca, Linnaeus, 1758) y el cachalote (Physeter catodon Linnaeus, 1758) (e.g., Ashford et al. 1996; Purves et al. 2004; Söffker et al. 2015; Tixier et al. 2010, 2015). Sin embargo, existe poca información disponible sobre la magnitud de estas interacciones en aguas chilenas. Aunque las interacciones entre la pesquería de bacalao y cetáceos en Chile son evidentes (e.g, Moreno et al. 2003; Hucke-Gaete et al. 2004; Rubilar et al. 2012), la ausencia de estudios sobre la ecología y biología de las especies de cetáceos involucrados no permite comprender los procesos biológicos y operacionales en estas interacciones, carencia que a nuestro juicio limita el desarrollo de estrategias de mitigación e implementación de nuevas tecnologías. El objetivo de este trabajo fue 
revisar y resumir la información existente en el país sobre el conocimiento actual que se disponen de las especies involucradas con la pesquería de este recurso bentónico denominado $D$. eleginoides, y del desarrollo de esta interacción.

\section{METODOLOGÍA}

Se realizó una búsqueda bibliográfica de antecedentes biológicos obtenidos en el país sobre el bacalao de profundidad, la orca y el cachalote, complementándolo con información publicada de otras áreas de su distribución. La información se organizó en dos categorías de bases de datos: informes técnicos y publicaciones científicas nacionales e internacionales. En cada una de estas bases de datos se extrajo la información referente a aspectos poblacionales (distribución) y biológicos/ ecológicos (rango de buceo, alimentación, reproducción y estructura social), así como de algunos aspectos operacionales relevantes, historia de las capturas de bacalao de profundidad y del conocimiento disponible acerca de las interacciones. Esta información fue sintetizada destacando aquellos resultados más importantes en cada tópico.

\section{RESULTADOS Y DISCUSIÓN}

\section{Ecología del bacalao de profundidad en Chile}

La información recopilada sobre la biología y ecología del bacalao de profundidad muestra que la mayoría de los estudios se han realizado en áreas de pesca en torno a islas que bordean el Frente Circumpolar Antártico, ubicadas en los sectores antárticos Atlántico e Índico, siendo menor las investigaciones en aguas sobre el talud y plataforma continental de Sudamérica (Chile y Argentina). Como es mencionado por Ferrada et al. (2008), los informes técnicos se concentran principalmente en los indicadores pesqueros, tales como captura, desembarque, esfuerzo y rendimiento de pesca y distribución espacio temporal; mientras los que se concentran en los indicadores biológicos, analizan la estructura de talla y composición de edad de las capturas de la pesquería de bacalao de profundidad desarrollada por la flota artesanal al norte del paralelo $47^{\circ} \mathrm{S}$ y la flota industrial que opera entre los $47^{\circ} \mathrm{S}$ y $57^{\circ} \mathrm{S}$. Además, se encuentran los estudios de evaluación de stock que basados en la información de los estudios de monitoreo estiman biomasa, abundancia y recomiendan las cuotas totales permisibles de bacalao de profundidad.

\section{Distribución}

El bacalao de profundidad se distribuye exclusivamente en el hemisferio sur $y$ en profundidades de 500 a 2000 metros (Arana, 2009), aunque la presencia de individuos adultos se ha registrado hasta los 2500 metros, en cañones submarinos de Chile (Salas et al. 1987; Young et al. 1996; Prenski, 2000). En el país, la especie habita preferentemente sobre el talud continental y su distribución batimétrica es inversamente proporcional a la latitud, encontrándose cerca del nivel de la plataforma en el sur y cerca de 2000 metros en el norte (Murillo et al. 2008). Los individuos de menor tamaño se encontrarían en aguas más someras, mientras que aquellos de mayor longitud se distribuirían en aguas más profundas (Moreno, 1991; Young et al. 1999; Ferrada et al. 2008).

\section{Alimentación}

La alimentación del bacalao de profundidad es variada, y dependiente de la latitud, profundidad y estado de desarrollo del individuo (Cassia \& Perrotta; 1996, García de la Rosa et al. 1997; Prenski, 2000; Murillo et al. 2008). Los ítems dietarios comprenden en su mayoría a organismos pertenecientes a las comunidades demersal y mesopelagial (Prenski, 2000). El bacalao de profundidad es considerado un carnívoro de tipo carcinófago e ictiófago, siendo las principales presas los peces (Cassia \& Perrotta, 1996; García de la Rosa et al. 1997; Prenski, 2000; Murillo et al. 2008). En Chile, los ítems dietarios están conformados por merluza del Pacífico (Merluccius gayi Guichenot, 1848), merluza de cola (Macruronus magellanicus Günther, 1873), sardina común (Sardinops sagax Jenyns, 1842), jurel del Pacífico sur (Trachurus murphyi Nichols, 1920), peje rata (Coelorhynchus sp. Gioma, 1809) y merluza azul (Antimora rostrata Günther, 1878), entre otros. Además, consume cefalópodos 
del orden Teutoidea (calamares) y Octopoidea (pulpos), crustáceos decápodos e incluso condrictios (Oyarzún \& Campos, 1987; Arrizaga et al. 1996; Oyarzún et al. 2003; Murillo et al. 2008). En la plataforma argentina, el canibalismo también ha sido informado para peces adultos en profundidades mayores a 800 m (Prenski, 2000). Además, Zhikov \& Krivoruchko (1990) observaron en especímenes de aguas antárticas una dieta eurifágica durante el primer año de vida, convirtiéndose luego en un típico predador de amplio espectro de alimento.

\section{Reproducción}

Es un pez relativamente longevo, con una fecundidad relativamente baja y comportamiento gregario, aunque se ha informado que los cardúmenes se separan por sexo, edad y talla (Young et al. 1992; Rubilar \& Moreno, 1998; Oyarzún et al. 2003). Recientes estudios en Chile, muestran que la especie presenta actividad reproductiva solo en la zona más austral, al sur del paralelo $47^{\circ} \mathrm{S}$, siendo sincrónica y ocurriendo en aguas menos profundas durante los meses de julio a octubre alcanzando su máxima expresión en septiembre (Young et al. 1992, 1999; Ferrada et al. 2008; Arana, 2009; Rubilar \& Zuleta, 2011). Así, la detección de una sola área principal de reproducción en la zona sur-austral, sugiere la existencia de una sola unidad poblacional de bacalao de profundidad en aguas chilenas. La madurez sexual en ambos sexos sería alcanzada aproximadamente a los 60-89,9 cm de longitud con una talla media de maduración en machos a $81 \mathrm{~cm}$ de longitud (nueve años de edad) y en hembras a $89 \mathrm{~cm}$ de longitud (11 años de edad; Arana, 2009). Estas tallas serían relativamente más cortas que aquellas estimadas previamente por Moreno et al. (1997; 105-117 cm), Young et al. (1999; 128.7 cm) y Oyarzún et al. (2003; 78-117 $\mathrm{cm})$ para el área geográfica de Chile y similares con aquellas de Argentina (Cassia \& Perrotta, 1996, 80-95 cm; Prenski \& Almeyda, 2000, 76-87 $\mathrm{cm})$. El número de óvulos varía entre 250.000 a medio millón por hembra (Arana, 2009; Rubilar \& Zuleta, 2011). Los óvulos una vez fecundados, ascienden paulatinamente hasta los $500 \mathrm{~m}$ de la columna de agua en el talud continental (Moreno et al. 1997; González, 2003), donde ocurriría la eclosión. La proporción sexual al sur de los $47^{\circ} \mathrm{S}$ sería mayormente conformada por machos con excepción de los meses de agosto y noviembre (Young et al. 1996, 1998; Arana, 2009), mientras que al norte de ese mismo paralelo, la proporción sexual se invierte siendo mayor para hembras en relaciones de 2,1:1 (Aris et al. 1999) e incluso de 3:1 para la zona de la I y II Regiones del país (Oliva et al. 1999).

No existen a la fecha trabajos de investigación en Chile referidos a la presencia de larvas y postlarvas de la especie. Para el Atlántico Sur, Ciechomski \& Weiss (1976) mencionan la captura de postlarvas en profundidades de entre 100-490 metros entre las latitudes $53^{\circ}-54^{\circ} 30^{\circ} \mathrm{S}$ de la plataforma patagónica argentina. En aguas de islas Falkland/Malvinas, Ehrlich et al. (1999) mencionan la presencia de larvas entre los 100200 metros de profundidad durante primavera; mientras que en las Georgia del Sur, larvas y postlarvas se encontrarían en la capa pelágica (North, 2002).

\section{Arte de pesca e historia de capturas}

Las artes de pesca involucradas en la captura del bacalao de profundidad han evolucionado en el tiempo. A inicios de la pesquería se usaban largas líneas de palangre español, espinel japonés o sistema mustad. Debido a que los rendimientos de pesca fueron disminuyendo y la acción depredadora de los cetáceos fue en aumento, se generó la necesidad de buscar mecanismos más efectivos para la captura y así mismo proteger los peces capturados. Actualmente, en el país se utiliza el palangre español modificado en conjunto con la cachalotera o sistema chileno (Rubilar et al. 2012) (Fig. 1).

La cachalotera fue una tecnología chilena adaptada del sistema español de palangre de pesca artesanal para implementarla en el sector industrial (Moreno et al. 2008). Este artefacto, consistente de una red en forma de cono flotante y ligada a la línea madre de la cual penden verticales de 15-20 $\mathrm{m}$, separadas cada una por $40 \mathrm{~m}$, en cuyo extremo inferior penden los reinales con los respectivos anzuelos y un peso. Así el funcionamiento de la cachalotera permite que se deslice a lo largo de la vertical, cubriendo los anzuelos cuando el arte 


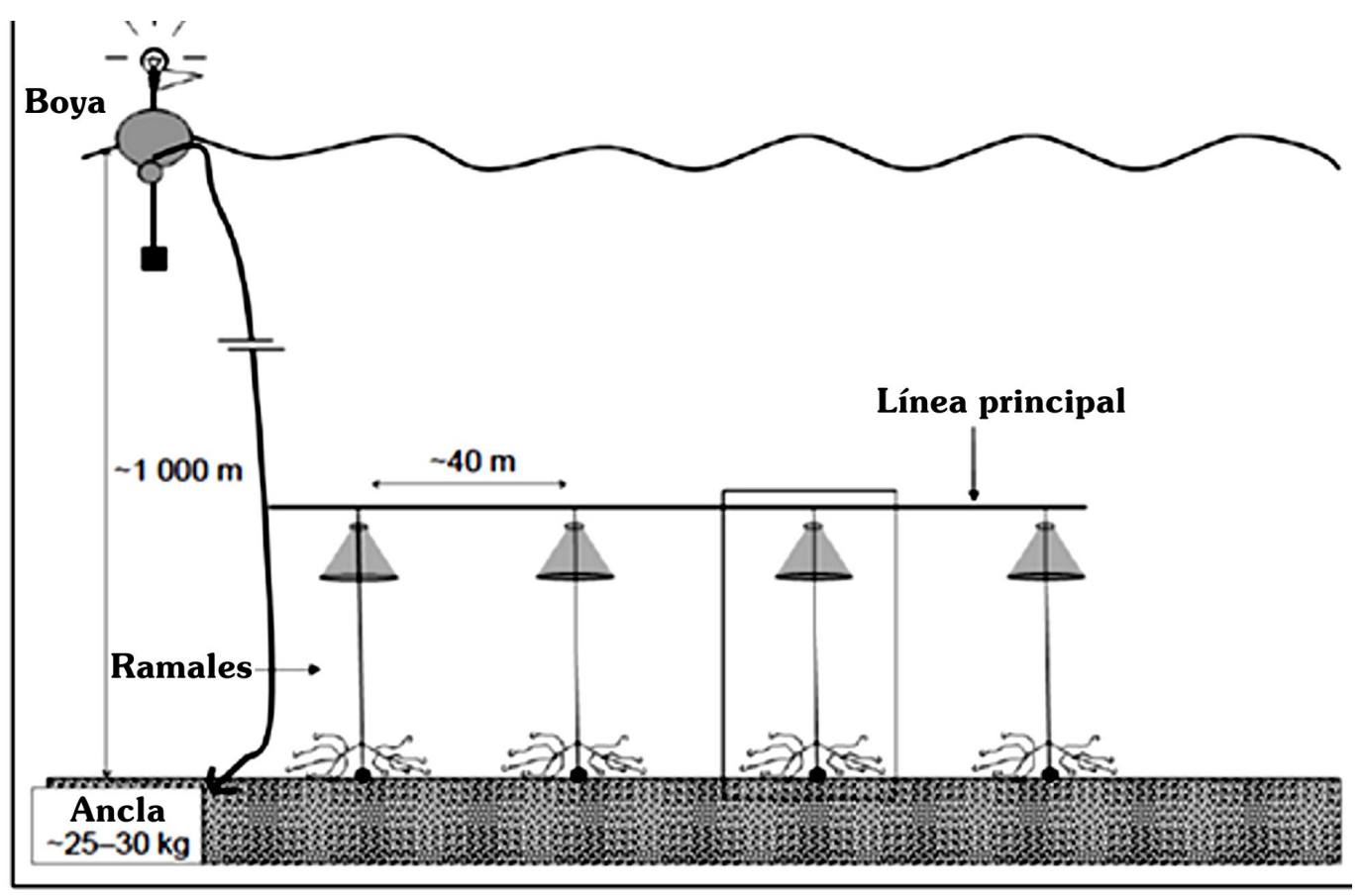

Fig. 1. Sistema español modificado con cachalotera para la pesca demersal de bacalao de profundidad. Tomado y modificado de Moreno et al. (2008).

de pesca es izado, protegiendo de esa manera los peces capturados (Fig. 1). Esta adaptación ha permitido disminuir significativamente la tasa de depredación por parte de los cachalotes, pero no el de las orcas, quienes han aprendido a tomar los peces capturados levantando la cachalotera, entre otros comportamientos aprendidos.

\section{Capturas}

El bacalao de profundidad constituye un recurso de importancia económica en Chile. Ésta pesquería se inicia en aguas del centro del país como pesca exploratoria en 1955 (González, 1962). A lo largo de la costa chilena operan dos tipos de pesquerías, una artesanal al norte del paralelo $47^{\circ} \mathrm{S}$ y que no tiene cuotas de capturas, y una pesquería industrial al sur del mismo y que está basada en un Régimen de Pesquerías con asignación de permisos extraordinarios de pesca, según la cuota anual de captura. La primera opera principalmente sobre la estructura juvenil de la población, mientras que la segunda lo hace sobre edades mayores (Young et al. 1999; Ferrada et al. 2008).
El bacalao de profundidad comenzó a ser explotado comercialmente en Chile por la flota artesanal en la década del 70, en la zona centro del país. La pesca de este recurso se extendió rápidamente hacia el norte y principalmente hacia el sur, donde se fueron detectando los mejores rendimientos (Lemaitre et al. 1991). La evolución de los desembarques muestra que las capturas de este nototénido en aguas chilenas ha presentado una tendencia de caída gradual desde el año 1992, cuando se capturaron cerca de 30.828 toneladas, a la fecha (Fig. 2). Las mayores capturas antes del año 1991 y después de 1998 es explicado mayormente por el aporte del sector artesanal; sin embargo desde el año 2000 el desembarque artesanal también comenzó a registrar una caída gradual. En los inicios de la serie, el desembarque estuvo representado principalmente por la actividad de la VIII Región, mientras que después de 1998, estaría representada por la actividad en la X Región (Valdivia y Quellón principalmente) (Quiroz, 2014). Aunque la flota industrial operaba en baja escala antes del año 1991 con desembarques inferiores a 5.000 ton, ésta tuvo un notorio aumento en las capturas en el área denominada licitada $\left(47^{\circ}-57^{\circ}\right.$ 

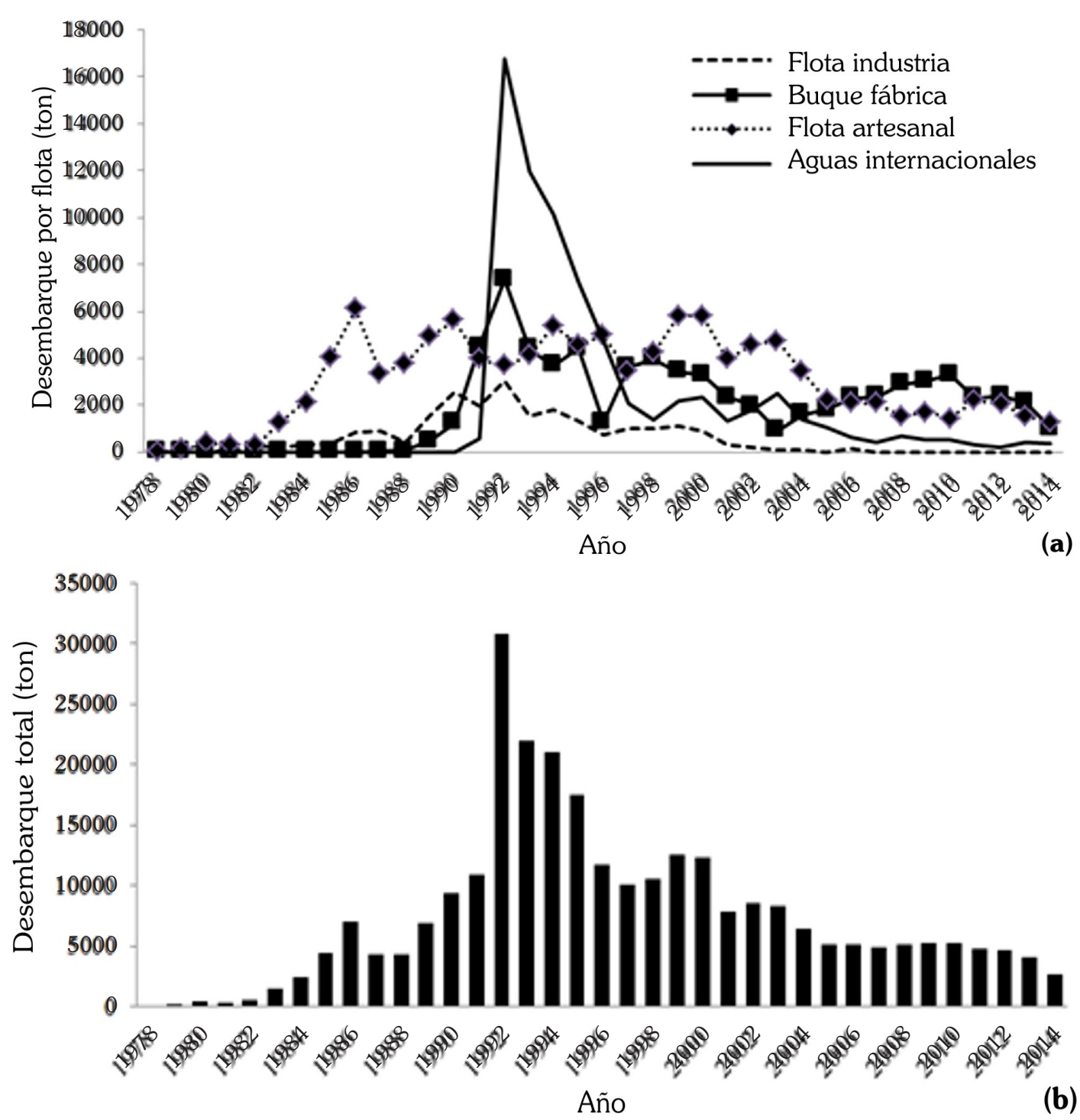

Fig. 2. Desembarques de bacalao de profundidad (Dissostichus eleginoides) por tipo de flota (a) y total (b) en Chile, según anuarios de Sernapesca.

S) entre los años 1992 y 1995 contribuyendo con la mayor parte del desembarque total en Chile con alrededor de $87,3 \%$. Sin embargo, el desembarque de éste sector también registra una fuerte caída, llegando a una relativa estabilización entre los años 2005 y 2006 (Ferrada et al. 2008, Quiroz, 2014). En los últimos años las capturas presentan una leve tendencia al alza (Quiroz, 2014).

La flota artesanal de Chile desarrolla sus actividades en tres zonas de pesca definidas (Oyarzún et al. 2003). Estas zonas son: Zona 1 desde los $32^{\circ} \mathrm{S}$ a $36^{\circ} \mathrm{S}$, Zona 2 entre $36^{\circ} \mathrm{S}$ y $40^{\circ} \mathrm{S}$, y la Zona 3 localizada entre los $40^{\circ} \mathrm{S}$ y $47^{\circ} \mathrm{S}$. A su vez, dentro de estas zonas existen focos de pesca discretos o "caladeros" en los cuales las capturas son marcadamente mayores. Aunque el rendimiento de la pesca artesanal del bacalao de profundidad a nivel país muestra una tendencia descendente desde 1998, en los puertos principales de la X Región el desembarque se ha mantenido constante debido a que las embarcaciones de esta zona incursionarían al sur de los $47^{\circ} \mathrm{S}$ (Ferrada et al. 2008).

Por su parte, en los inicios de la pesquería 
que desarrolla la flota industrial espinelera, la permanencia en el área licitada era de forma más extensa en tiempo (septiembre a febrero del año siguiente), pero con el correr de los años la flota industrial ha tendido a una disminución gradual debido a la disminución de las cuotas otorgadas. El caladero que principalmente aportaba a la captura y al rendimiento de pesca de bacalao de profundidad por la flota industrial ha sido el caladero más austral, localizado al sur del paralelo $55^{\circ} \mathrm{S}$ y limitando con Argentina (Ferrada et al. 2008).

La estructura de talla del desembarque artesanal de bacalao se ha caracterizado por una fuerte componente de ejemplares juveniles $(90 \%)$ y una talla media de $90 \mathrm{~cm}$ (Ferrada et al. 2008); sin embargo, Quiroz (2014) señala que se requiere la revisión de la información de estructura en edades o tallas. La estructura de talla de la captura de bacalao por la flota industrial ha mostrado tener históricamente un fuerte componente de fracciones adultas de la población (Ferrada et al. 2008). En la serie histórica de la pesquería industrial también se aprecia una constante predominancia de machos con respecto a las hembras. Para las hembras, las capturas informadas entre los años 1996-2000, el mayor componente estuvo representado por grupos de edad mayores (XI y XIV años) respecto a los machos. En el año 2001, la moda se ubicó en el grupo de edad VI, aunque también tuvo mayor presencia en los grupos de edad mayor. Entre los años 2004 al 2007 se presentó una estructura con mayor fracción de jóvenes que el resto de la serie. Se debe mencionar que esta serie de estructura del desembarque en número por grupo de edad comprendió análisis de escamas desde su inicio hasta el año 2006 y análisis de otolitos desde el año 2007 al presente, siendo este más preciso que el utilizado a través de escamas (Quiroz, 2014).

En 1996, la Subsecretaría de Pesca estableció una veda reproductiva entre los meses de junio y agosto entre el paralelo $53^{\circ} \mathrm{S}$ y $57^{\circ} \mathrm{S}$ (D.S. Exento $\left.\mathrm{N}^{\circ} 273,6 / 12 / 1996\right)$. No obstante, durante esta veda no se aprovechó de obtener información sobre la reproducción y y ocurrencia de la actividad de desove del recurso. Actualmente, el recurso se encontraría en estado de "Sobreexplotación" y con su biomasa desovante por debajo del $20 \%$ de la biomasa original (Inf. Tec. N²03-2014).

\section{Conocimiento de la orca y} el cachalote en Chile

La información disponible sobre distribución y conocimiento biológico y/o ecológico de la orca en Chile es escasa. A la fecha, estas son dos notas presentadas en Congreso (Canto et al. 1990; Marcotte et al. 2009), dos reportes presentados en el Comité Científico de la Comisión Ballenera Internacional (Mikhalev et al. 1981, Dahlheim et al. 1982) y cuatro trabajos publicados (AguayoLobo et al. 1998; Capella et al. 1999, 2014; Häusserman et al. 2013). Nuestro conocimiento sobre la interacción con la pesquería del bacalao en aguas chilenas proviene de dos trabajos publicados (Hucke-Gaete et al. 2004; Moreno et al. 2008) y tres informes (Moreno et al. 2003; Rubilar \& Zuleta, 2011; Rubilar et al. 2012). Por su parte, el conocimiento sobre los cachalotes es mayor; aunque varios de los trabajos se remontan al período ballenero (e.g., Clarke, 1958; AguayoLobo, 1963; Clarke et al. 1964, 1976, 1980, 1988, 2011). Posterior al período ballenero, resalta el trabajo de Aguayo-Lobo et al. (1998) quienes realizan un resumen de la información existente, y otro de Coakes \& Whitehead (2004) para las aguas del norte del país. Al igual que las orcas, información sobre la interacción con la pesquería del bacalao se encuentra en los mismos trabajos e informes mencionados anteriormente.

\section{Orcinus orca}

\section{Distribución}

La orca presenta una distribución cosmopolita y habita aguas costeras y pelágicas de todo el mundo (Rice, 1998). Hasta la fecha, la presencia de orcas en aguas del mar territorial de Chile, ha sido informada desde el sur de Arica (18 50 'S), hasta el mar de Bellingshausen (70²3`S), incluyendo el archipiélago de Juan Fernández (Aguayo-Lobo et al. 1998, Capella et al. 1999). No se conoce la existencia de grupos residentes en el país aunque algunos individuos fotoidentificados han sido re-avistados durante y entre años en aguas australes (Häussermann et al. 2013; Capella et al. 2014). Cuatro formas geográficas de orcas han sido reconocidas en el Hemisferio Sur, 
denominadas morfotipos A, B, C y D (Pitman \& Ensor, 2003; Pitman et al. 2011) (Fig. 3), de los cuales dos de estos morfotipos, los tipos A y D han sido registrados en aguas jurisdiccionales del país.

El primer morfotipo corresponde a la orca que se conoce a nivel mundial. Presenta la típica coloración blanca y negra sobre el cuerpo. El macho alcanza los 9 metros de longitud y presenta una aleta dorsal alta y normalmente de forma triangular. La hembra alcanza una longitud cercana a los 7 metros, y posee una aleta dorsal falcada y generalmente terminada en punta o suavemente redondeada. Ambos sexos poseen un parche ocular de tamaño medio orientado paralelamente al eje del cuerpo. Detrás de la aleta dorsal presenta una montura de coloración blanquecina que puede ser abierta o cerrada. Basado en estas características, la gran mayoría de los registros que se tienen para Chile corresponderían a este morfotipo (e.g., Aguayo-Lobo et al. 1998; Häussermann et al. 2013; Capella et al. 1999, 2014).

El morfotipo D fue documentado por primera vez en el año 2011 (Pitman et al. 2011). Este morfotipo sería el más pequeño de los cuatro morfotipos descritos. Posee una coloración blanca y negra similar al morfotipo A, pero la forma de la cabeza es más globosa semejante al calderón negro (Globicphala spp. Traill, 1809), y la montura detrás de la aleta dorsal es difusa. Es notoriamente distinguible de los otros morfotipos por presentar una diminuta mancha ocular orientada paralelamente al eje horizontal del cuerpo. La aleta dorsal es estrecha, terminada en punta angosta y generalmente bastante angulada, especialmente evidente en machos adultos Este morfotipo ha sido documentado con una distribución circumglobal en aguas subantárticas entre las latitudes $40^{\circ} \mathrm{S}$ y $60^{\circ} \mathrm{S}$. Solo dos avistamientos han sido documentados en el paso Drake entre los $58^{\circ} 39^{\prime} \mathrm{S}$ y $60^{\circ} 10^{\prime} \mathrm{S}$ (Pitman et al. 2011). No obstante, relatos de capitanes de barco que operan en la pesquería industrial del bacalao de profundidad, señalan su presencia al sur de los $56^{\circ} \mathrm{S}$, aunque esos relatos deben ser aún confirmados.

Los otros dos morfotipos presentarían una distribución circumpolar, siendo el tipo B especialmente común en el área de la península Antártica, mientras que el tipo $\mathrm{C}$ habita latitudes
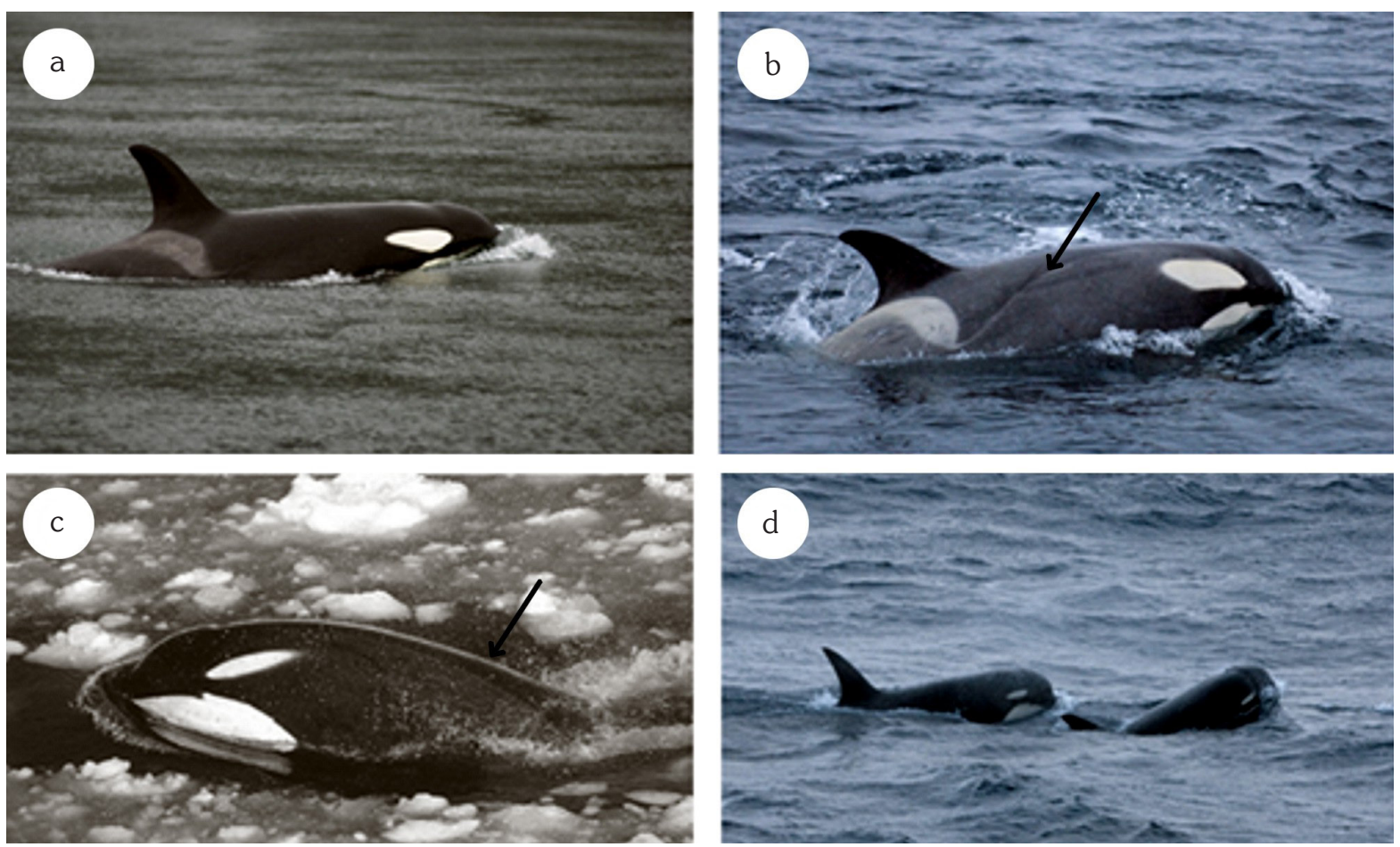

Fig. 3. Morfotipos de orcas del Hemisferio Sur. (a) Morfotipo A, (b) Morfotipo B, (c) Morfotipo C y (d) Morfotipo D.

Fotografía: J. Acevedo, Jorge Plana y tomada de Pitman \& Ensor (2003). Con flechas se indica la presencia de la capa dorsal. 
más altas entre los hielos y aguas costeras siendo especialmente común en la región del mar de Ross (Pitman \& Ensor, 2003). Movimientos de algunos individuos del tipo $\mathrm{B}$ han sido documentados hasta los $30^{\circ} \mathrm{S}-37^{\circ} \mathrm{S}$ frente a Uruguay y Brasil (Durban \& Pitman, 2012), y por tanto no se puede descartar su posible presencia ocasional frente a la costa Pacífica de Sudamérica. Externamente, los morfotipos $\mathrm{B}$ y $\mathrm{C}$ son animales algo más pequeños que el morfotipo A. Aunque presentan el mismo patrón de coloración, estos son más grisáceos que negros, mientras que las áreas blancas son usualmente de coloración amarillenta causada por diatomeas. Otras características distinguibles son el tamaño y orientación del parche ocular. En el tipo $\mathrm{B}$, el parche ocular es de gran tamaño (cerca del doble que el tipo A) y orientado horizontalmente, mientras que en el tipo $\mathrm{C}$ la mancha ocular es más pequeña y orientada cerca de $45^{\circ}$ al eje horizontal del cuerpo. Además, ambos morfotipos presentan una capa dorsal gris oscura que se proyecta desde la montura localizada detrás de la aleta dorsal, y la montura es generalmente de forma cerrada. Esta capa dorsal está ausente en los morfotipos A y D (Pitman \& Ensor 2003; Pitman et al. 2011).

\section{Rango de buceo}

La orca generalmente nada en o cerca de la superficie (20 metros) y usualmente bucean por no más de 30 segundos. Sin embargo, pueden alcanzar profundidades superiores a los 100 metros o más cuando están en búsqueda de alimento. La máxima profundidad registrada para una orca ha sido de 767 metros y una duración máxima de 14 minutos (Baird et al. 1998, 2005; Sivle et al. 2012; Reisinger et al. 2015).

\section{Alimentación}

La dieta de la orca es muy variada y en el hemisferio Sur incluye especies de tiburones, mantarrayas, teleósteos, pinnipedios, tortugas marinas, pequeños cetáceos y aves (e.g., Gaskin, 1982; Jefferson et al. 1991; Visser et al. 2010). En el país, el conocimiento sobre la biología y ecología de las orcas es escasa, y más aún sobre sus hábitos alimentarios. La poca información existente proviene de observaciones ocasionales realizadas en el sistema de canales y fiordos patagónicos y fueguinos y no se conoce la dieta en aguas oceánicas. Los avistamientos en aguas interiores señalan la depredación regular sobre el lobo marino común (O. flavescens Shaw, 1800) y en menor extensión sobre el lobo fino sudamericano (A. australis Zimmermann, 1783). Depredación ocasional sobre algunas especies de aves marinas como cormorán imperial (Phalacrocorax atriceps King, 1828), gaviota dominicana (Larus dominicanus Linchtenstein, 1823) y pingüinos de Magallanes (Spheniscus magellanicus Forster, 1781) y peces (posiblemente merluza austral o congrio dorado) han sido informados (Capella et al. 1999, 2014; Häussermann et al. 2013). Anecdóticamente, también se menciona un ataque sobre un ejemplar de ballena sei (Balaenoptera borealis Lesson, 1828) en los canales nor-patagónicos y la muerte pero no consumo de petrel buceador (Pelecanoides magellanicus Lacepede, 1799) en los canales fueguinos (Capella et al. 2014).

Basado en la literatura, habrían diferencias sustanciales en la dieta de acuerdo a los morfotipos descritos anteriormente. Así, la orca tipo A sería un predador generalista (Pitman et al. 2011), lo cual concuerda con las observaciones anteriores para Chile. La orca tipo B se alimentaría preferentemente de focas y lobos marinos aunque ballenas minkes y pingüinos también son parte de la dieta en aguas antárticas (Pitman \& Ensor, 2003; Visser et al. 2008; Pitman \& Durbam, 2010, 2012), mientras que la tipo C consumiría principalmente bacalao antártico (Dissostichus mawsoni) (Pitman \& Ensor, 2003). Se desconoce la dieta de la orca tipo $\mathrm{D}$, pero se ha sugerido que podría incluir peces (Pitman et al. 2011). Reisinger et al. (2015) basados en datos de buceos de alta profundidad $(767 \mathrm{~m})$ registrados en algunos individuos de orca en aguas de isla Marion, sugieren que podrían estar depredando sobre cefalópodos que migran verticalmente $y$ quizás sobre bacalao de profundidad.

\section{Reproducción}

En el país no se dispone de ningún estudio sobre reproducción de la especie. La literatura señala que las orcas son extremadamente longevas, con hembras alcanzando longevidades máximas de 
hasta 90 años de edad (Olesiuk et al. 1990). La madurez reproductiva ocurre a los 10 años para muchas hembras y producen una cría cada 5-6 años a lo largo de su vida reproductiva (desde 10 a 46 años) (Olesiuk et al. 2005). Los machos alcanzan la madurez sexual cercano a los 15 años (Olesiuk et al. 2005) pero continúan en crecimiento hasta alcanzar la madurez física cerca de los 21 años (Ford, 2002). La gestación parece a ser de 15-18 meses y la edad de destete cercana a los 2 años de vida (Olesiuk et al. 2005). Los nacimientos pueden ocurrir en cualquier mes aunque es dependiente del ecotipo de orca. Así por ejemplo, los nacimientos en orcas de tipo residentes (hemisferio norte) ocurren con mayor frecuencia entre octubre $y$ marzo (Ford, 2002).

\section{Estructura social}

La organización social básica es matriarcal, compuesta de hembras líderes, los hijos y nietos. Esta composición parece ser estable en el tiempo, pero se ha detectado cambios a escalas de tiempo más largas (Capella et al. 2014). En Chile y basado en la información de avistamientos que se disponen, se observan generalmente grupos pequeños (promedio $=4-5$ animales), aunque grupos mayores de hasta aproximadamente 60 animales han sido ocasionalmente registrados (Häussermann et al. 2013, Capella et al. 2014). Los tamaños de grupos aquí señalados son similares a los informados para otras regiones del mundo, y grandes grupos probablemente envuelven agregaciones temporales de unidades sociales más estables (Ford, 2002).

\section{Physeter macrocephalus}

Esta especie es el representante más grande del Suborden Odontoceti. El macho puede llegar a medir 18 m y las hembras adultas no más de 12,3 $\mathrm{m}$. El peso de los machos adultos fluctúa entre 45-69 toneladas y el de las hembras entre 15-20 toneladas (Clarke, 1956). El cuerpo es lateralmente comprimido y con una gran cabeza que alcanza $1 / 4$ a $1 / 3$ de la longitud total. Presenta una aleta dorsal baja, generalmente triangular, seguida de una serie de protuberancias en el pedúnculo caudal. La aleta caudal es ancha y triangular con bordes lisos que se muestra en el momento de la inmersión (Clarke, 1956).

\section{Distribución}

El cachalote es una especie cosmopolita y habita todas las aguas profundas del globo (Clarke, 1956) desde el trópico hasta el límite del pack-ice en ambos hemisferios (Whitehead, 2002). Solamente los machos se encuentran en altas altitudes, mientras que las hembras y crías se localizan en latitudes bajas y medias (Whitehead, 2002). En nuestro país se le ha registrado desde Arica hasta las aguas antárticas, y desde la costa hasta los $110^{\circ} \mathrm{W}$ incluyendo el archipiélago de Juan Fernández, islas Desventuradas, Pascua y Salas y Gómez (Aguayo-Lobo et al. 1998).

\section{Rango de buceo}

El cachalote es uno de los cetáceos conocidos por bucear a grandes profundidades, alcanzando los 3.200 metros. No obstante, los rangos de profundidad durante la alimentación comúnmente son de $400-900 \mathrm{~m}$, permaneciendo entre 30 y 45 minutos sumergidos. Los machos alcanzarían mayores profundidades de buceos que las hembras, permaneciendo hasta 120 minutos (Whitehead, 2002).

\section{Alimentación}

El cachalote se alimenta en toda la columna de agua, y especialmente en aguas demersales, consumiendo calamares y peces. Para alimentarse realiza inmersiones más allá de los 800-1000 metros de profundidad (Mathews, 1938; Clarke, 1956; Clarke et al. 1988; Whitehead, 2003). En Chile, la dieta ha sido informada exclusivamente para aguas del norte y centro-sur siendo principalmente de calamares (Histioteuthis sp, Chiroteuthis sp, Octopoteuthis sp, Dosidicus gigas, Vampyroteuthis infemalis, Ancistrocheirus lesueuri) (Clarke et al. 1976, Clarke et al. 1988). Restos de peces (> 1\%) fueron también registrados así como dos especies de grandes centollas (Clarke et al. 1988). No se dispone de datos sobre alimentación para la región austral del país. Aunque su principal ítem alimentario se 
basa en cefalópodos mesopelágicos de pequeño y gran tamaño, en algunas zonas el cachalote consume numerosas especies de peces demersales y mesopelágicos (Whitehead, 2002) incluyendo entre ellas a Dissostichus eleginoides en el Océano Atlántico y D. mawsoni en el Pacífico (Kawakami, 1980).

\section{Reproducción}

Es una especie polígama, donde el macho puede atender a más de 15 hembras maduras (Mathews, 1938). La vida de las hembras es eminentemente social, mientras que los machos adultos son solitarios (Whitehead, 2003). Los estudios sobre reproducción en Chile se remontan a la década de 1950 y 1960 llevados a cabo durante las investigaciones balleneras realizadas en Chile y Perú. Esos resultados muestran que las hembras maduran sexualmente a la longitud de 8,2-8,6 metros y 6,5 años (Clarke et al. 1964, Clarke et al. 1980, Clarke et al. 2011) siendo altamente fértiles entre los 15 y 35 años de edad (Clarke et al. 2011). La madurez física es alcanzada a los 11,2 metros de longitud y 33 años de edad (Clarke et al. 2011). Los machos alcanzan la madurez sexual a los 13 m (Aguayo-Lobo, 1963) y una edad de 19 años (Clarke et al. 1994), la madurez social la logran a los 14-15 metros y 24 años de edad (Clarke et al. 1980; Clarke et al. 1994), mientras que la madurez física la alcanzan a los 16,5 metros de longitud y 43 años de edad. La duración de la preñez es cercana a los 17 meses (Clarke et al. 1964), el período de lactancia alcanza, en promedio, unos 19 meses y el período de reposo post-destete unos 12 meses, dando un intervalo de ciclo sexual de 4 años (Clarke et al. 2011). El período de apareamiento sería muy prolongado, desde abril a febrero, con muchas concepciones ocurriendo entre junio y diciembre, aunque el máximo se produciría en septiembre (Clarke et al. 1964; Clarke et al. 2011). La proporción sexual de los fetos sería cercana a la razón 1:1 (55,1\% machos y 44,9\% hembras) (Clarke et al. 2011).

\section{Estructura social}

La organización social básica es matriarcal, compuesta de hembras, machos y hembras jóvenes y crías (Whitehead, 2003). Los machos adultos son generalmente avistados solos, especialmente al sur de los $45^{\circ} \mathrm{S}$ (Mathews, 1938). En el norte de Chile, los tamaños promedio de grupos de hembras con inmaduros fueron cerca de 23 animales, mientras que el tamaño de la unidad social básica (hembras e inmaduros con permanente relación) fue de 11 animales. El grupo de machos adultos consistió en el $4 \%$ de la población, siendo avistados principalmente durante julio a septiembre (Coakes \& Whitehead, 2004).

Los cachalotes presentan una alta segregación sexual, con machos jóvenes dejando la unidad natal y moviéndose al igual que los machos adultos a regiones de altas latitudes (Best, 1979). Estos movimientos a altas latitudes por parte de los machos muestran pocos signos de estructura social comparados con las hembras e inmaduros que permanecen en latitudes bajas y medias (Letteval et al. 2002). En este sentido, los tamaños de grupos informados en las áreas de pesca austral del bacalao de profundidad variaron de 1 a 18 animales durante el período 2002-2003 (Moreno et al. 2003), observándose en septiembre la mayor cantidad de ejemplares (Hucke-Gaete et al. 2004). Durante el período 2006-2012, los tamaños de grupos han variado de 1 a 9 animales, siendo los individuos solitarios y parejas los más persistentes en el tiempo (Rubilar \& Zuleta, 2011, Rubilar et al. 2012). Estos últimos autores también señalan una variación estacional en los tamaños de grupos, siendo septiembre/octubre los meses en que se observa la mayor amplitud de grupos y la mayor cantidad de los grupos más grandes.

\section{Conocimiento de la interacción}

entre la pesquería del bacalao de profundidad y cetáceos en Chile

Los cetáceos, en general, consumen presas que no tienen valor comercial y cuando consumen aquellas que sí lo tienen, lo hacen en lugares apartados donde no hay pesca significativa por el hombre. En consecuencia, la competencia es pequeña y no causan grandes daños económicos a escala global. Sin embargo, también existen áreas donde la sobreposición de las pesquerías y los cetáceos ocurre generando problemas serios e importantes para ambos. Las interacciones entre 
cachalotes y orcas con la pesquería del bacalao de profundidad ocurren desde hace varios y años en varias localidades del mundo (Ashford et al. 1996; Purves et al. 2004; Söffker et al. 2015; Tixier et al. 2010, 2015), y nuestro país no es ajeno a aquella situación.

El primer estudio realizado en aguas nacionales sobre esta interacción se enmarcó principalmente en la problemática de la interacción con el cachalote en la región austral del país (Moreno et al. 2003). En ese entonces, información anecdótica y de observadores científicos sugerían que las interacciones eran frecuentes, con consecuencias negativas para la pesquería y para los cetáceos (Salas et al. 1987). Este estudio mostró evidencias que el cachalote dominaba por número y frecuencia (60\%) durante las operaciones pesqueras, siendo responsable de la mayor frecuencia de observaciones de pesca dañada, mientras que la orca (tipo A) solo alcanzaba el $10 \%$ de las observaciones (Moreno et al. 2003, Hucke-Gaete et al. 2004). Estas observaciones fueron similares a aquellas informadas en la pesquería del bacalao de profundidad en isla Georgia del Sur durante las temporadas 2000-2002 (Purves et al. 2004; Clark et al. 2010) donde la frecuencia de cachalotes era mayor con respecto a las orcas. La tasa promedio de interacción operacional (depredación) informada para Chile fue estimada en $3 \%$ y cuando ambas especies estuvieron presentes bajó a niveles cercanos a $0 \%$, concluyendo que los efectos de la depredación ocasionada por ambas especies de cetáceos sobre la biomasa del stock de bacalao de profundidad serían mínimos, y en consecuencia no habrían argumentos para aseverar que había una competencia severa con la pesquería por un mismo recurso, ni tampoco que la tendencia declinante del rendimiento pesquero fuera atribuible al efecto de la depredación por cetáceos. No obstante, la pérdida monetaria total presumiblemente causada a la flota industrial parecía ser de consideración (media = USD \$92.684). Sin embargo, esta cifra debe ser tomada con precaución debido a que ésta se encuentra probablemente maximizada debido al bajo rendimiento que tiene la pesquería actualmente (Moreno et al. 2003; Hucke-Gaete et al. 2004).

Dada a la continua acción depredadora de los cetáceos, particularmente del cachalote, se generó la necesidad de buscar mecanismos para proteger los peces capturados. Así, la adaptación de la cachalotera en el arte de pesca durante el año 2006 bajó a la mitad la tasa de depredación con respecto al año 2002 (Moreno et al. 2008). Sin embargo, las actividades pesqueras que se han realizado posteriormente, indican que la cachalotera no estaría funcionando con las orcas, como si lo hace con el cachalote. Por otra parte, en la última década también se ha detectado un cambio de dominancia en la composición de cachalotes/ orcas presentes en esta pesquería, siendo hoy las orcas las que dominan en presencia y frecuencia de interacción en la región austral de Chile (Rubilar et al. 2012). Este cambio composicional también ha sido informado en otras regiones del Hemisferio Sur (e.g., Roche et al. 2007; Clark \& Agnew, 2010; Tixier et al. 2010; Söffker et al. 2015).

Entre los años 2009-2012, nueva información sobre la interacción entre la pesquería del bacalao y cetáceos, incluyendo estimación de la tasa de depredación, fue generada por el Centro de Estudios Pesqueros (CEPES). Uno de esos resultados es que las mayores frecuencias de avistamiento de orcas y cachalotes ocurrieron al sur de los $55^{\circ} \mathrm{S}$ con las mayores abundancias ocurriendo durante invierno-primavera (Rubilar \& Zuleta, 2011; Rubilar et al. 2012). Además, las orcas (tipo A) serían especialmente dominantes en el área de las islas Diego Ramírez. Los mismos autores también informan que la depredación a partir de los vestigios de peces muestra una variación mensual, con una mayor tasa de depredación en los meses finales de invierno y principios de primavera, con valores máximos estimados en un $10 \%$. Además, esta fluctuación tendría una alta correlación con la variación estacional de la cantidad de orcas y cachalotes en las zonas de pesca. Sin embargo, esta estimación estaría subestimada dado a que se basa en la observación de vestigios de bacalao que llegan a bordo.

En aguas de las islas Georgias del Sur, Clark y Agnew (2010) documentan que durante el período 2006 al 2009, la captura del bacalao de profundidad se afectó en un $18 \%$ cuando los cachalotes estuvieron presentes y solo un $4 \%$ en presencia de orcas. Sin embargo, la cantidad de peces removidos fue mucho mayor con orcas que con cachalotes. Similarmente, Söffker et al. (2015) 
señalan que la depredación de orcas sobre el arte de pesca fue significativamente más alta que aquella de cachalotes, con tasas de captura que disminuyeron entre un $63 \%$ y $97 \%$, cuando las orcas estuvieron presentes. La alta tasa de depredación se concentra en ciertas áreas y mayormente entre los meses de mayo y agosto (Söffker et al. 2015).

En aguas de isla Crozet, la tasa de depredación solo por cachalotes, solo por orcas y cuando ambas especies están presentes alcanza actualmente valores de $25 \%, 22 \%$ y $53 \%$, respectivamente (Guinet et al. 2014), siendo mucho más altas que aquellas estimadas previamente para el período 2003-2005 (Roche et al. 2007) y 2003-2008 (Tixier et al. 2010). Al igual que en las islas Georgias del Sur, la pérdida de peces fue mucho mayor en presencia de orcas que de cachalotes (Guinet et al. 2014). Interesantemente, Guinet \& Tixier (2011) y Tixier et al. (2016) informan que la interacción de orcas con la pesquería del bacalao de profundidad en aguas de isla Crozet está dada por los morfotipos A y D; mientras que en islas Georgia del Sur posiblemente por el morfotipo B además del A (Clark \& Agnew, 2010). Aunque en aguas chilenas solo se ha documentado hasta la fecha la interacción del morfotipo A, las formas $\mathrm{B}$ y $\mathrm{D}$ no pueden ser descartados y la revisión de fotografías de orcas interactuando con la pesquería del bacalao de profundidad al sur de los $55^{\circ} \mathrm{S}$ debe ser siempre realizada y analizada por expertos.

Finalmente, las medidas de mitigación más efectivas para ambas especies, a nuestro juicio, se encontrarán a futuro, cuando nuestro conocimiento sobre el comportamiento de estas dos especies de cetáceos sea mayor. Por tanto, estudios a mediano y largo plazo deben ser iniciados en este contexto, en el marco de un programa de investigación colaborativo, eficiente y sinérgico, con el apoyo del Gobierno a través de la Subsecretaría de Pesca y con la Industria Pesquera con sus buques pesqueros, a fin de llevar a cabo estudios capaces de buscar y encontrar las mejores soluciones de mitigación de los efectos ecológicos y económicos-sociales de las mencionadas interacciones.

\section{AGRADECIMIENTOS}

Los autores agradecen a la Asociación de Investigadores del Museo de Historia Natural de Río
Seco, a la Dirección del Instituto Antártico Chileno (INACH) y a la Dirección del Centro de Estudios del Cuaternario Fuego Patagonia y Antártica (Fundación CEQUA) por las facilidades otorgadas para la realización de este trabajo. A Eduardo Infante por la organización e invitación a participar en el 1er Seminario de Bacalao, en Septiembre del año 2015 y a COLTO Depredation Workshop, en Marzo del 2016, así como a los colegas de la Universidad de Magallanes que nos han invitado gentilmente para publicar este trabajo en una serie especial dedicado a esta especie.

\section{LITERATURA CITADA}

Aguayo-Lobo, A. (1963). Observaciones sobre la madurez sexual del cachalote macho (Physeter catodon L.), capturado en aguas chilenas. Montemar (Cont. Rev. Biol.). Valparaíso, 11(3), 99-125.

Aguayo-Lobo, A., Torres, D., \& Acevedo, J. (1998). Mamíferos marinos de Chile. I. Cetacea. Serie Científica Inach, (48), 19159.

Aguayo-Lobo, A. (1999). Los cetáceos y sus perspectivas de conservación. Estudios Oceanológicos, 18, 35-43.

Arana, P. M., \& Vega, R. (1999). Exploratory for Dissostichus spp. in the Antarctic Region (Subarea 48.1, 48.2 and 88.3). CCAMLR Science, 6, 1-17.

Arana, P. (2009). Reproductive aspects of Patagonian toothfish (Dissostichus eleginoides) off Southern Chile. Latin American Journal of Aquatic Research, 37(3), 381-349.

Angliss, R., \& DeMaster, D. (1997). Differentiating serious and non-serious injury of marine mammals taken incidental to commercial fishing operations: Report of the Serious Injury Workshop. Silver Spring, MD, USA.

Aris, L., Gálvez, P., Céspedes, R., Olguín, A., Miranda, H., Adarme, L., \& Young, Z. (1999). Estudio biológico pesquero de los recursos demersales explotados por la flota artesanal en el litoral de la III y IV Regiones. Informe Final FIP-IFOP 97-17, 97 p + Anexo.

Arrizaga A., Fuentealba, M., Yañez, V., \& Oyarzún, 
C. (1996). Observaciones sobre la alimentación de Dissostichus eleginoides Smitt 1898. (Perciformes, Nototheniidae) en el sur de Chile. Gayana Oceanologica, 4(1), 13 - 19.

Ashford, J. R., Rubilar, P. S., \& Martin, A. R. (1996). Interactions between cetaceans and longline fishery operations around South Georgia. Marine Mammal Science, 12(3), $452-457$.

Baird, R. W., Dill, L. M., \& Hanson, M. B. (1998). Diving behavior of killer whales. Abstracts of the World Marine Mammal Science Conference, Monaco.

Baird, R. W., Hanson, M. B., \& Dill, L. M. (2005). Factors influencing the diving behavior of fish-eating killer whales: sex differences and diel and interannual variation in diving rates. Canadian Journal of Zoology, 83, 257-267.

Best, P. B. (1979). Social organization in sperm whales, Physeter macrocephalus. En $\mathrm{H}$. E. Winn \& B. L. Olla (Eds.), Behavior of marine animals Vol 3 (pp. 227-289). New York: Plenum Press.

Canto J., Cárdenas, J. C., \& Yañez, J. (1990). Distribución y tamaño grupal de Orcinus orca (Linnaeus, 1758) en Chile. En: Resumen $4^{\mathrm{a}}$ Reunión de trabajo de especialistas en mamíferos acuáticos de América del Sur. Valdivia, Chile.

Capella, J., Gibbons, J., \& Vilina, Y. (1999). The killer whale, Orcinus orca (Delphinidae) in Chilean waters between Arica and Cabo de Hornos. Anales del Instituto de la Patagonia, 27, 63-72.

Capella, J., Abramson, J. Z., Vilina, Y., \& Gibbons, J. (2014). Observations of killer whales (Orcinus orca) in the fjords of Chilean Patagonia. Polar Biology, 37, 15331539.

Cassia, M. C., \& Perrotta, R. G. (1996). Distribución, estructura de tallas, alimentación y pesca de la merluza negra (Dissostichus eleginoides Smith, 1898) en un sector del Atlántico Sudoccidental. INDEP Informe Técnico 9.

Ciechomski, J. D., \& Weiss, G. (1976). Desarrollo y distribución de postlarvas de róbalo
Eleginops maclovinus (Valenciennes, 1830) Dollo 1904, de la merluza negra Dissostichus eleginoides Smitt 1898 y de las nototenias Notothenia spp., Pisces, Notothenidae. Pysis, 35(19), 115-125.

Clark J., \& Agnew, D.J. (2010). Estimating the impact of depredation by killer whales and sperm whales on longline fishing for toothfish (Dissostichus eleginoides) around South Georgia. CCAMLR Science, 17, 163-178.

Clarke, R. (1956) Sperm whales of the Azores. Discovery Reports, 28, 237-298.

Clarke, R., Aguayo, L., A. \& Paliza, O. (1964). Progress report on sperm whale research in the Southeast Pacific Ocean. Norsk Hvalfangst-Tidende, 53, 297-302.

Clarke, M., MacLeod N., \& Paliza, O. (1976). Cephalopod remains from the stomachs of sperm whales caught off Peru and Chile. Journal of Zoology, 180(4), 477-493.

Clarke, R., Paliza, O., \& Aguayo L., A. (1980). Some parameters and an estimate of the exploited stock of sperm whales in the Southeast Pacific between 1959 and 1961. Reports of the International Whaling Commission, 30, 289-305.

Clarke, R., Paliza, O., \& Aguayo L., A. (1988). Sperm whales of the Southeast Pacific. Part IV. Fatness, food and feeding. Investigations on Cetacea, 21, 53-195.

Clarke, R., Paliza, O., \& Aguayo L., A. (1994). Sperm whales of the Southeast Pacific. Part VI. Growth and breeding in the male. Investigations on Cetacea, 25, 93-224.

Clarke, R., Paliza, O., \& Van Waerebeek, K. (2011). Sperm whales of the Southeast Pacific. Part VII. Reproduction and growth in the female. Latin American Journal of Aquatic Mammals, 10(1): 8-39.

Coakes, A., \& Whitehead, H. (2004). Social structure and mating system of sperm whales off northern Chile. Canadian Journal of Zoology, 82, 1360-1369.

Dahlheim M. E., Leatherwood, S., \& Perrin, W. F. (1982). Distribution of killer whales in the warm temperate and tropical eastern Pacific. Report of the International Whaling Commission, 32, 647-653. 
Durban, J. W., Pitman, R. L. (2012). Antarctic killer whales make rapid, round-trip movements to subtropical waters: evidence for physiological maintenance migrations? Biological Letters, 8, 274-277.

Ehrlich, M. D., Sánchez, R. P., Ciechomski, J. D., Machinandiarena, L., \& Pájaro, M. (1999). Ichtyoplankton composition, distribution and abundance on the southern Patagonian shelf and adjacent waters. In: Reproductive habitat, biology and acoustic biomass estimates of the southern blue whiting (Micromesistius australis) in the sea off southern Patagonia. INDEP Doc. Cient., 5, 37-65.

Ferrada, S., Hernández, C., Canales-Aguirre, C., Aedo, G., San Martin, M., \& Astete, S. (2008). Unidades poblacionales del bacalao de profundidad. Informe Final Proyecto $\mathrm{N}^{\circ}$ 2006-41 del Fondo de Investigación Pesquera. Universidad de Concepción.

Ford, J. K. B. (2002). Killer whale Orcinus orca. En W. F. Perrin, B. Würsig \& J. G. M Thewissen (Eds.), Encyclopedia of marine mammals (pp. 669-676). San Diego: Academic Press.

García de la Rosa, S. B, Sánchez, F., \& Figueroa, D. (1997). Feeding ecology of Dissostichus eleginoides in two areas: Patagonian shelf and Georgias Islands. CCMLAR Science, 4, 105-124.

Gaskin, D. E. (1982). The Ecology of Whales and Dolphins. Heinemann, London and Exeter.

González, G. O. (1962). Pesca experimental con espinel de profundidad. Memoria de Prueba para optar al título de Técnico Pesquero. Universidad Católica de Valparaíso, Chile

González N. E. F. (2003). Interacciones entre la pesquería artesanal del bacalao de profundidad, Dissostichus eleginoides, y cetáceos en aguas del Centro $\left(28^{\circ} \mathrm{S}\right)-$ Sur $\left(46^{\circ} \mathrm{S}\right)$ de Chile. Tesis de Licenciatura, Facultad de Ciencias del Mar. Universidad de Valparaíso, Valparaíso, Chile.

Guinet, C., \&Tixier, P. (2011). Crozet Killer Whales: A Remote But Changing Environment. Journal of American Cetacean Society, 40, 33-38.
Guinet, C, Tixier, P., Gasco, N., \& Duhamel, G. (2014). Long-term studies of Crozet Island killer whales are fundamental to understanding the economic and demographic consequences of their depredation behavior on the Patagonian toothfish fishery. ICES Journal of Marine Science, 72(5), 1587-1597.

Häussermann, V., Acevedo, J., Forsterra, G., Bailey, M., \& Aguayo-Lobo, A. (2013). Killer whales in Chilean Patagonia: additional sightings, behavioural observations, and individual identifications. Revista de Biología Marina y Oceanografía, 48(1), 73-85.

Hucke-Gaete, R., Moreno, C. A., \& Arata, J. (2004). Operational interactions of sperm whales and killer whales with the Patagonian toothfish industrial fishery off southern Chile. CCAMLR Science, 11, 127-140.

Jefferson, T. A., Stacey, P. J., Baird, R. W. (1991). A review of killer whale interactions with other marine mammals: predation to coexistence. Mammal Review, 21, 151180.

Kaschner, K., \& Pauly, D. (2005). Competition between marine mammals and fisheries: Food for thought. En D.J. Salem \& A. N. Rowan (Eds.), The state of the animals III: 2005 (pp.95-117). Washington, DC: Humane Society Press.

Kawakami, T. (1980). A review of sperm whale food. Science Report Whales Research Institute 32, 199-218.

Lemaitre, C., Rubilar, P., Gebauer, P., \& Moreno, C. A. (1991). Regional catch analysis of longline fishery of Dissostichus eleginoides (Pisces: Nototheniidae) in Chile. Document WG-FSA-91/10. CCAMLR, Hobart, Australia.

Letteval, E., Richter, C., Jaquet, N., Slooten, E., Dawson, S., Whitehead, H., Christal, J., \& McCall Howard, P. (2002). Social structure and residency in aggregations of male sperm whales. Canadian Journal of Zoology, 80, 1189-1196.

Marcotte, M., Häussermann, V., Biro, J., \& Försterra, G. (2009). The role of the silent 
warden: Trophic interactions between killer whales and sea lions in Chilean Patagonia. En: Congreso de Ciencias del Mar. Talcahuano, Chile.

Matthews, L. H. (1938) The sperm whale, Physeter catodon. Discovery Reports, 17, 93-168.

Mikhalev, Y. A., Ivashin, M. V., Savusin, V. P., \& Zelenaya, F. E. (1981). The distribution and biology of killer whales in the Southern Hemisphere. Report of the International Whaling Commission, 31, 551-565.

Moreno, C. A. (1991). Hook selectivity in the longline fishery of Dissostichus eleginoides (Nototheniidae) off the Chilean coast. En Selected Scientific Papers, 1991 (SC-CAMLR-SSPI8). CCAMLR, Hobart, Australia (pp. 107-119).

Moreno, C. A., Rubilar, P. S., \& Zuleta, A. (1997). Ficha técnica del bacalao de profundidad. Document WG-FSA-9 7/42. CCAMLR, Hobart, Australia.

Moreno, C. A., Hucke-Gaete, R., \& Arata, J. (2003). Interacción de la pesquería del bacalao de profundidad con mamíferos $y$ aves marinas. Informe Final Proyecto FIP N²001-31. Fondo de Investigación Pesquera, Valparaíso, Chile.

Moreno, C. A., Castro, R., Mújica L. J., \& Reyes, P. (2008). Significant conservation benefits obtained from the use of a new fishing gear in the Chilean Patagonian toothfish fishery. CCAMLR Science, 15, 79-91.

Murillo C., Oyarzún, C., \& Fernández, I. (2008). Variación latitudinal y estacional en la dieta de Dissostichus eleginoides Smitt, 1898 (Perciformes: Notothenidae) en ambientes profundos de la costa centro-sur de Chile. Gayana, 72(1), 94-101.

North, A. W. (2002). Larval and juvenile distribution and growth of Patagonian toothfish around South Georgia. Antarctic Science, 14, 25-31.

Northridge, S. P. (1984). World review of interactions between marine mammals and fisheries. Fisheries Technical Paper No. 251, Food and Agriculture Organization of the United Nations.

Northridge, S. P., \& Hofman, R. J. (1999). Marine mammal interactions with fisheries. En
J. R. Twiss Jr. \& R. R. Reeves (Eds.), Conservation and management of marine mammals (pp. 99-119). Washington, D.C.: Smithsonian Institution Press.

Olesiuk, P., Bigg, M., \& Ellis, G. (1990). Life history and population dynamics of resident killer whales (Orcinus orca) in the coastal waters of British Columbia and Washington State. Report of the International Whaling Commission, Special, 209-243.

Olesiuk, P. F., Ellis, G. M. \& Ford, J. K. B. (2005). Life history and population dynamics of northern resident killer whales in British Columbia. Research document 2005/45, Ottawa, Canada: Canadian Science Advisory Secretariat, Fisheries and Oceans Canada.

Oliva, J., Young, Z., \& Martínez, F. (1999). Caracterización de las pesquerías de cojinova del norte y bacalao de profundidad en la I y II Regiones. Informe Final FIPIFOP 97-18.

Oyarzún, C., \& Campos, P. (1987). Dissostichus eleginoides (Smitt, 1898); consideraciones sobre su determinación taxonómica e implicancias biogeográficas (Pisces, perciformes, Nototheniidae). Revista de Biología Marina, 23(2), 173-192.

Oyarzún, C., Gacitúa, S., Araya, M., Cubillos, L., Salamanca, M., Pino, C., Galleguillos, R., ...Lamilla, J. (2003). Asignación de edades y crecimiento de bacalao de profundidad. Informe Final, Proyecto FIP 2001-17.

Oyarzún, C., Gacitúa, S., Araya, M., Cubillos, L., Galleguillos, R., Pino, C., Aedo, G.,...Lamilla, J. (2003). Monitoreo de la pesquería artesanal de bacalao de profundidad entre la VIII y XI regiones. Informe Final, Proyecto FIP N²001-16.

Pávez, P, Yañez, E., Salas, N., Tarky, W., Rojas, P., \& Flores, H. (1983). Estudio del bacalao de profundidad (Dissostichus eleginoides) como recurso pesquero en la región de Valparaíso. Evaluación biológica, tecnológica y económica. Estudios y Documentos Universidad Católica de Valparaíso, Facultad de Recursos Naturales, Escuela de Ciencias del mar. 
Valparaíso, Chile. $N^{\circ} 1,83-136$.

Pitman, R. L., \& Ensor, P. (2003). Three different forms of killer whales in Antarctic waters. Journal of Cetacean Research Management, 5, 131-139.

Pitman, R. L., \& Durban, J. W. (2010). Killer whale predation on penguins in Antarctica. Polar Biology, 33, 1589-1594.

Pitman, R. L., \& Durban, J. W. (2012). Cooperative hunting behavior, prey selectivity and prey handling by pack ice killer whales (Orcinus orca), type B, in Antarctic Peninsula waters. Marine Mammal Science, 28(1), 16-36.

Pitman, R. L., John, D., Michael, G., Guinet, C., Jorgensen, M., Olson, P., Plana, J.,... Towers, J. (2011). Observations of a distinctive morphotype of killer whale (Orcinus orca), type D, from subantarctic waters. Polar Biology, 34, 303-306

Prenski, L. B. (2000). Merluza negra (Dissostichus eleginoides). Pesquería de Argentina 1997-1999, 81-92.

Prenski, L. B., \& Almeida, S. (2000). Some biological aspect relevant to Patagonian toothfish (Dissostichus eleginoides) exploitation in the Argentine exclusive economic zone and adjacent ocean sector. Frente Marítimo, A, 103-124.

Purves, M. G., Agnew, D. G., Balguerías, E., Moreno, C. A., \& Watkins, B. (2004). Killer whale (Orcinus orca) and sperm whale (Physeter macrocephalus) interactions with longline vessels in the Patagonian fishery at South Georgia, South Atlantic. CCAMLR Science, 11, 111-126.

Quiroz, J. C. (2014). Investigaciones del estatus y posibilidades de explotación biológicamente sustentables en bacalao de profundidad, año 2014. Segundo Informe Final, Instituto de Fomento Pesquero, Valparaíso, Chile.

Reisinger, R. R., Keith, M., Andrews, R. D., \& de Bruyn, P. (2015). Movement and diving of killer whales (Orcinus orca) at a Southern Ocean Archipelago. Journal of Experimental Marine Biology and Ecology, 473, 90-102.

Rice, D. W. (1998). Marine Mammals of the World:
Systematics and Distribution. Society for Marine Mammalogy, Special Publication, 4, 231.

Roche, C., Gasco, N., Duhamel, G., \& Guinet, C. (2007). Marine mammals and demersal long line fishery interactions in Crozet and Kerguelen Exclusive Economic Zones: Anassessment of the depredation level. CCAMLR Science Series, 14, 67-82.

Rubilar, P., \& Moreno, C. (1998). Ficha Técnica del Bacalao de Profundidad Dissostichus eleginoides, Smitt 1898. Universidad Austral de Valdivia, Chile.

Rubilar, P. S., \& Zuleta, A. (2011). Bases para un programa colaborativo de monitoreo científico en la pesquería del bacalao. Informe Final Pesca de Investigación Bacalao 2010. Centro de Estudios Pesqueros (CEPES S.A.).

Rubilar, P. S., Moreno, C. A., \& Zuleta, A. (2012). Monitoreo pesquería-dependiente de la abundancia del bacalao e interaión de los mamíferos con la pesca en el área licitada. Informe Final Pesca de Investigación Bacalao 2011. Centro de Estudios Pesqueros (CEPES S.A.).

Salas, R., Robotham, H., \& Lizama, G. (1987). Investigación del Bacalao en la VIII región. Intendencia región del Bío-Bío, Instituto de Fomento Pesquero.

Sivle, L. D., Kvadsheim, P. H., Fahlman, A., Lam F. P. A, Tyack, P. L., \& Miller, P. J. O. (2012). Changes in dive behavior during naval sonar exposure in killer whales, longfinned pilot whales, and sperm whales. Frontiers in Physiology 3, Article 400.

Söffker, M., Trathan, P., Clark, J., Collins, M.A., Belchier, M., \& Scott, R. (2015). The impact of predation by marine mammals on Patagonian toothfish longline fisheries. PLoS ONE, 10(3), e0118113.

Tixier, P., Gasco, N., Duhamel, G., Viviant, M., Authier, M., \& Guinet, C. (2010). Interactions of Patagonian toothfish fisheries with Killer and Sperm whales In the Crozet Islands Exclusive Economic Zone: an assessment of depredation levels and insights into possible mitigation strategies. CCAMLR Science Series, 17, 
179-195.

Trites, A., Villy, C., \& Pauly, D. (1997). Competition between fisheries and marine mammals for prey and primary production in the Pacific Ocean. Journal of Northwestern Atlantic Fisheries, 22: 173-187.

Visser, I. N., Smith, T. G., Bullock, I. D., Green, G. D., Carlsson, O. G. L., \& Imberti, S. (2008). Antarctic peninsula killer whales (Orcinus orca) hunt seals and a penguin on floating ice. Marine Mammal Science, 24, 225-234.

Visser, I. N., Zaeschmar J., Halliday, J., Abraham, A., Ball, P., Bradley, R., \& Daly, S. (2010) First record of predation on false killer whales (Pseudorca crassidens) by killer whales (Orcinus orca). Aquatic Mammals, 36, 195-204.

Whitehead, H. (2002). Sperm whales Physeter macrocephalus. En W. F. Perrin, B. Würsig \& J. G. M. Thewissen (Eds.), Encyclopedia of marine mammals (pp. 1165-1172.). San Diego: Academic Press,.

Whitehead, H. (2003). Sperm whales: social evolution in the ocean. Chicago: University of Chicago Press.

Young, Z., Zuleta, A., Robotham, H., Aguayo, M., \& Cid, L. (1992). Evaluación del stock de bacalao de profundidad entre las latitudes $47^{\circ}$ y $57^{\circ} \mathrm{S}$. Informe Técnico IFOP/ SUBPESCA.

Young, Z., Robotham, H., \& Gili, R. (1996). Evaluación de la pesquería y del stock de bacalao de profundidad al sur del paralelo $47^{\circ}$ L.S. Informe Final FIP-IFOP 94-10.

Young, Z., González, H., \& Gálvez, P. (1998). Análisis de la pesquería de bacalao de profundidad en la zona sur-austral. Informe Final FIP-IFOP 96-40.

Young, Z., Oliva, J., Olivares, A., \& Díaz, E. (1999). Aspectos reproductivos del recurso bacalao de profundidad en la I a X Regiones. Informe Final Proyecto FIP 97-16.

Zhikov, V. V., \& Krivoruchko, V. M. (1990). On the biology of the Patagonian toohfish, Dissostichus eleginoides of the Antarctic part of the Atlantic. Voprosy Ikhtiologii, 30(5), 861-864. 